\title{
Prevalence of Cryptosporidium Species and Other Intestinal Parasites Among HIV Infected Patients at LAUTECH Teaching Hospital, Osogbo, Nigeria
}

\author{
Akinseye Janet Fumilayo", Adedokun Samuel Adeyinka², Adewuyi Isaac Kayode ${ }^{2}$, \\ Agunlejika Richard Adedokun ${ }^{2}$, Thomas Hosea Zagi ${ }^{3}$, Ayuba Sunday Buru ${ }^{4}$ \\ ${ }^{1}$ Department of Medical Laboratory Science, College of Medicine and Health Sciences, Afe Babalola University, Ado-Ekiti, Nigeria \\ ${ }^{2}$ Department of Microbiology and Parasitology, School of Medical Laboratory Sciences, Obafemi Awolowo University Teaching Hospital, \\ Ile-Ife, Nigeria \\ ${ }^{3}$ Department of Medical Laboratory Science, Faculty of Health Sciences, College of Medicine, Kaduna State University, Kaduna, Nigeria \\ ${ }^{4}$ Genomic Research Lab, Department of Medical Microbiology and Parasitology, Faculty of Basic Clinical Sciences, College of Medicine, \\ Kaduna State University, Kaduna, Nigeria
}

Email address:

akinseyejanet@gmail.com (A. J. Fumilayo) sunday.buru@kasu.edu.ng (A. S. Buru)

\section{To cite this article:}

Akinseye Janet Fumilayo, Adedokun Samuel Adeyinka, Adewuyi Isaac Kayode, Agunlejika Richard Adedokun, Thomas Hosea Zagi, Ayuba Sunday Buru. Prevalence of Cryptosporidium Species and Other Intestinal Parasites Among HIV Infected Patients at LAUTECH Teaching Hospital, Osogbo, Nigeria. Biomedical Sciences. Vol. 7, No. 1, 2021, pp. 25-28. doi: 10.11648/j.bs.20210701.15

Received: January 22, 2021; Accepted: February 1, 2021; Published: March 4, 2021

\begin{abstract}
Background: Cryptosporidiosis though an opportunistic infection in HIV infected patients, increases the mortality and morbidity to the diseases by its effect suppression of the immune system, our quest is to highlights the clinical correlation of diarrhea in immunocompromised individuals and the economic losses associated with managing infected individuals. Methods: Stool samples were collected from 96 subjects. Their personal information regarding their names, sex, age was also obtained. The stool samples were processed for non-opportunistic parasite using the direct and formol-ether concentration method. For Cryptosporidium, Modified Ziehl-Neelsen Technique was used. A total of ninety-six confirmed HIV individuals were enrolled for this study. Results: The mean age of the patients was 24.7 years with the youngest been 3 years old and the eldest 58 years old. 77 (74\%) were females while 22 (23\%) were males. Table 1 shows the general characteristics of the HIV subjects recruited into the study. The distribution of intestinal parasites detected in patient's stool is shown in Table 2 . Of the 96 stool samples examined, intestinal parasites were seen in 87 samples, while 9 samples showed absence of parasites. The diagnosed parasitic infections were: Cryptosporidium spp (54.2\%), A. lumbricoides (9.4\%), Hookworm (5.2\%), E. histolytica $(3.1 \%)$, S. stercoralis (1\%) and Teania spp (1\%). Double parasitic infections with Cryptosporidium were observed in 13 (13.5\%) HIV/AIDS patients stool samples. Regarding age and sex distribution of the participants, there were 74 (77\%) females and $22(23 \%)$ males. Males (68.2\%) were more infected than females $(55.4 \%)$ but the difference was not statistically significant. Table 3 shows relationship between intestinal parasitic infection and diarrhea in the HIV/AIDS patients. 45 (86.5\%) of patients with Cryptosporidium infection presented with diarrhea. Conclusion: The association between diarrhea and Cryptosporidium infection was statistically significant. It may be concluded that in HIV infected patients, both opportunistic intestinal parasitic infections are prevalent.
\end{abstract}

Keywords: Cryptosporidium Spp, HIV/AIDS, Intestinal Parasites

\section{Introduction}

The connection between certain enteric parasites and human immunodeficiency virus (HIV) infection is well documented. Gastrointestinal involvement in HIV/AIDS is almost universal, and significant diseases occur in $50-90 \%$ of immunocompromised and malnourished patients [1] while diarrhea can be presenting manifestation or a life threatening complication in HIV patients sometimes during the course of 
the disease [2]. The etiology for such diarrhea could either be parasitic, bacterial, fungal, enteric virus or HIV itself [3]. Several species of protozoa have been associated with acute and chronic diarrhea in HIV diseases. These include: Crptosporidium parvum, Isospora belli, Microsporidia species, Giardia intestinalis, Entamoeba histolytica, Cyclospora species, Blastocysis hominis and Dientamoeba fragilis [2]. Nematodes like strongloides stercoralis can also cause diarrhea and overwhelming infestation in patients with variety of immunosuppresive disorders including HIV/AIDS $[4,5]$. Helminths, including hookworm, Ascaris lumbricoides, Teania saginata and Trichuris trichura, are considered non opportunistic, except on rare occasions $[6,7]$.

Opportunistic infections are caused by either organisms of low or no virulence which are nonpathogenic for a person with intact immune system. The infection caused by known pathogen in such immunocompromised patients may present in different form like increased virulence, recurrence, multidrug resistant, or atypical presentation [8]. Intracellular intestinal protozoans Cryptosporidium parvum, Clyclospora cayetanensis, Isospora belli, and the microsporidia are most frequent opportunistic in patients with acquired immunodeficiency syndrome (AIDS) and are often the major cause of uncontrollable, debilitating diarrhea [9]. Cryptosporidium spp is one of the enteric microorganisms most commonly associated with persistent diarrhea (PD) and is associated with wasting in immunologically compromised individuals involving mostly people with HIV/AIDS [10]. This parasite causes morbidity and mortality in AIDS patient worldwide, and these outcomes would be expected to be appreciably higher in developing countries due to higher prevalence of infection in the general population. About 42 million people are infected globally with HIV while sub-Saharan Africa account for more than half (29.4 million) of this number (10). In Nigeria, infection rate of HIV range between $4.9 \%$ and $5.8 \%$ [11].

Except for a few published cases, there is insufficient information on the prevalence and magnitude of the clinical importance of Cryptosporidium infection in HIV/AIDS patient in tropical and developing countries. Some studies in Africa suggested that the incidence of infection might be less as might be expected given the higher prevalence of immunosuppression due to HIV/AIDS in the continent [12]. However, this could be attributed to a lack of adequate diagnostic methods specific to these parasites in this part of the world of which research are currently ongoing [13, 14].

This study seeks to highlights the opportunistic significance of Cryptosporidiosis infection and clinical correlation of diarrhea in immunocompromised individuals, the economic losses associated with management of infection and persistent debilitating diarrhea experience by infected individuals as major concerns.

\section{Materials and Methods}

\subsection{Study Area}

The study was carried out at the HIV clinic of Ladoke
Akintola University of Technology (LAUTECH) Osogbo. Osogbo is the capital city of Osun State, Nigeria located in the rain forest zone. It is located between latitude $7^{\circ} 641 \mathrm{~N}$ and $4^{\circ} 341 \mathrm{E}$ with a human population of about 845,000 (National Census, 2006).

\subsection{Sample Collection and Analysis}

Stool samples were collected from consenting HIV patients attending the HIV clinic for routine checkup, drug collection and other clinical complaints. Clean sterile universal bottle was given to after being educated on how to properly collect the stool into it.

\subsection{Analysis of Stool Sample}

The stool was examined microscopically using direct saline and iodine preparation for the identification of trophozoites and cysts of protozoan parasites while the formal-ether concentration technique was used to identify cysts and ova of intestinal parasites. Ziehl-Neelsen staining was carried out for the identification of oocyst of Cyptosporidium spp as described by Cheesbrough, 2005.

\subsection{Direct Saline Preparation}

A piece of the feacal sample was mixed on the slide with physiological saline and iodine. The resulting suspension was sufficiently thin in order to transmit light easily. The sample was spread out and covered with a coverslip (N0.1) and examined microscopically at a magnification of x10 and x40.

\subsection{Modified Formal-ether Sedimentation Technique}

All stool samples, about $1 \mathrm{~g}$ of feaces was emulsified in $7 \mathrm{ml}$ of $10 \%$ formalin in a centrifuge tube. The suspension was strained through a brass wire sieve into a beaker to collect the filtrate. The filtrate was poured into a centrifuge tube and $3 \mathrm{ml}$ of ether as added. The tube was shaken vigorously by hand for one minute and then centrifuge immediately at $3000 \mathrm{rpm}$ for one minute. The layer of feacal debris was loosen with the aids of an applicator stick from the side of the centrifuge tube and inverted quickly to discard the supernatant. The sediment was resuspended by tapping the bottom of the tube. A drop of resuspended mixture was transferred onto a clean microscope slide and covered with a cover slip. This was examined microscopically using x10 objective lens, then $\mathrm{x} 40$ for confirmation and identification of parasites [12].

\subsection{Modified Ziehl-Neelsen Technique}

Ziehl-Neelsen staining was carried out as described by Cheesbrough, [15]. Smears were prepared from the sediment obtained by formol-ether concentration technique. The smears were air dried and fixed with methanol for 2 minutes. The fixed smears were stained with carbol fuschin for 15 minutes, and were washed with water. This was followed by decolourization with $3 \%$ acid alcohol for 10 seconds and washed immediately with water. The smears were counter 
stained with $0.5 \%$ methylene blue, and the slides were stood in a draining rack for the smears to dry. The stained smears were examined microscopically for oocysts, using x100 oil immersion objective lens to identify them.

\subsection{Statistical Analysis}

Test for statistical significance and association was done using T-test and Chi-square.

\section{Results}

A total of ninety-six confirmed HIV individuals were enrolled in this study. The mean age of the patients was 24.7 years with the youngest been 3 years old and the eldest was 53 years old. $74(77 \%)$ were females while $22(23 \%)$ were males. Table 1 shows the general characteristics of the HIV subjects recruited into the study.

The distribution of intestinal parasites detected in patient's stools is shown in Table 2. Of the 96 stool sample examined, intestinal parasites were seen in 87 samples, while 9 samples showed absence of parasites. The diagnosed parasitic infections were: Cryptosporidium spp (54.4\%), $A$. lumbroicodes (9.4\%), Hookworm (5.2\%). E. histolytica (3.1\%), S. stercoralis (1\%) and Teania spp (1\%). Double parasitic infections with Cryptosporidium were observed in $13(13.5 \%)$ HIV infected patients stool samples examined.

Regarding age and sex distribution of the participants, there were $74(77 \%)$ females and $22(23 \%)$ males. Males $(68.2 \%)$ were more infected than females $(55.4 \%)$, it equally shows the relationship between intestinal parasitic infection and diarrhea in HIV patients. $45(86.5 \%)$ of patients with Cryptosporidium infection presented with diarrhea.

Table 1. General Characteristic of Subjects.

\begin{tabular}{ll}
\hline Patients Characteristics & Number observed \\
\hline Number of subject studied & 96 \\
Sex: Male: Female & $22: 74$ \\
Mean age & $24.7 \mathrm{yrs}$ \\
Subjects positive for Cryptosporidium spp (\%) & $52(54.2 \%)$ \\
Subjects positive for other intestinal parasites $(\%)$ & $19(19.8 \%)$ \\
Co-infection of Cryptosporidium and other intestinal & $13(13.5 \%)$ \\
parasites (\%) & $60(62.5 \%$ \\
Number with diarrhea & \\
\hline
\end{tabular}

Table 2. Prevalence of intestinal parasites in HIV infected patients.

\begin{tabular}{ll}
\hline Parasite seen & AIDS Patients N=96 (\%) \\
\hline SINGLE & \\
Cryptosporidium spp & $55(54.2)$ \\
Ascaris lumbricoides & $9(9.4)$ \\
Hookworm & $5(5.2)$ \\
E. histolytica & $3(3.1)$ \\
S. stercoralis & $1(1.0)$ \\
Teania spp & $1(1.0)$ \\
DOUBLE INFECTION & \\
Cryptosporadium + A. lumbricoides & $4(4.2)$ \\
Cryptosporadium + Hookworm & $5(5.2)$ \\
Cryptosporadium + E. histolytica & $3(3.1)$ \\
Cryptosporadium + S. stecoralis & $1(1.0)$ \\
\hline
\end{tabular}

Table 3. Intestinal parasitic infection among HIV positive patients according to age.

\begin{tabular}{lllll}
\hline \multirow{2}{*}{ Age Range } & Male & Female & No. (\%) +ve & P-value \\
\cline { 2 - 5 } & No. Examined & No.(\%) +ve & No. Examined & $3(60)$ \\
20 & 3 & $2(67)$ & 5 & $22(63)$ \\
$21-30$ & 4 & $2(50)$ & 35 & $9(53)$ \\
$31-40$ & 5 & $4(80)$ & 26 & $6(86)$ \\
$41-50$ & 6 & $5(83)$ & 7 & $1(100)$ \\
$>50$ & 4 & $2(50)$ & 1 & $41(55.4)$ \\
Total & 22 & $15(68.2)$ & 74 & 0.0001 \\
\hline
\end{tabular}

\section{Discussion}

HIV infection and its health and economic consequence is a threat to people in sub-Saharan Africa [16]. It has also been associated with chronic diarrhea which is usually as a result of opportunistic parasitic infection. Intestinal parasitic infections are among the leading causes of morbidity and mortality in patients infected with HIV. Cryptosporidium spp is a well-established cause of diarrhea among HIV infected patients worldwide with prevalence of infection ranging from $3 \%$ in developed countries to $50 \%$ in developing countries [17]. An overall prevalence of $54.2 \%$ of Cryptosporidium infection was noted in HIV/AIDS patients in this study. The prevalence rate reported in this study is similar to what was previously reported in this area and other African countries [18].
The prevalence of $73.9 \%$ was recorded for overall intestinal parasitic infection among the HIV subjects. This is in line with the reports from other part of Nigeria. [19], observed a prevalence rate of $70.8 \%$ in Ilorin, Northern Nigeria, while [20], recorded prevalence rate of $89.5 \%$ in Lagos, Nigeria. Results of this study also reveal the trend in the occurrence of specific parasites in HIV positive persons in Osogbo, Nigeria. Ascaris Lumbroicodes, (9.4\%), Hookworm (5.2\%), Entameoba Histolytica (3.1\%), Teania spp (1\%) and Stronglyoides stercoralis (1\%). These findings are in agreement with those of Okodua et al., [21], who reported Hookworm (5.7\%), Entamoeba histolytica (5.7\%) and Strongyloides stercoralis (2.9\%). Although, Stongyloides stercoralis can cause overwhelming infection in HIV/AIDS patients, its detection among HIV patients in this study is also in agreement with study from Addis Abba (3.4\%) [22, 23].

While non opportunistic intestinal parasites infections are 
still an important problem in HIV-infected patients at any immunity level with or without the symptoms of diarrhea. With adequate medication, diarrhea as a result of immunosuppression will be tackled.

\section{Conclusion}

In conclusion, this study confirmed the widespread existence of Cryptosporidium spp in HIV/AIDS patients in Nigeria. Therefore, considering the severe and untreatable nature of Cryptosporidiosis and other opportunistic parasites should be done routinely for this category of patients, and control measures should be taken to achieve improved management among HIV/AIDS population.

Further study need to be done to characterize the species of Cryptosporidium as this will help to identify the pathogenic forms and increase our understanding of their genomic sequence. The use of Polymerase Chain Reaction technique in diagnosis may proof more efficient.

\section{References}

[1] Rumpa S, Bhoomika S, Sungtila TJ and Shwetank S. Prevalence of cryptosporidiosis in symptomatic immunocompetent children and comparative evaluation of its diagnosis by Ziehl-Neelsen staining and antigen detection techniques. Trop Parasitol. 2019 Jan-Jun; 9 (1): 18-22.

[2] Awole M, Gebre-selassie S, Kassa T, Kibru G. Prevalence of Intestinal parasites in HIV-1 infected adult patients in southwestern Ethiopia. Ethiop. J Health Dev 2003; 17 (1): 71 78 .

[3] Soave R, Framm SR. Agent of Diarrhoea. Med Clin. North Am. 1997; 81 (2): 427-447.

[4] Pollock RCG, Farthing MJG. Managing gastrointestinal infection in AIDS. Trop. Doc 1997; 28 (5): 238-241.

[5] Ambrioise-Thomas. Parasitic Diseases and immunodeficiency. Parasitology 2001; 122.

[6] Gompels MM, Todd J, Peters BS, Main J, Pinching AJ. Disseminated strongyloidiasis in AIDS: uncommon but important. AIDS 1991; 5: 329-332.

[7] Hung CC, Chen PJ, Hsieh SM, Wong JM, Fang CT, Chang SC Chen MY. Invasive amoebiasis: an emerging parasitic disease in patients infected with HIV in an area endemic for amoebic infection. AIDS 1999; 13: 2421-2428.

[8] Mulla SA, Manish, GP, Greeta V, Nazima M, Vikas D, Ratan KS. A study of opportunistic infection in HIV-Sero positive patients. Indian Journal of Community Medicine 2007; 32 (3): 208-209.

[9] Maggi P, Larocca A, Quarto M, Serio G, Brandonisio O, Angarano G. Effect of antiretroviral therapy on cryptosporidiosis and microsporidiosis in patients infected with human immunodeficiency virus type 1. Eur J Clin Microbiol Infect Dis 2000; 19: 213-217.
[10] James K, Tumwine Addy Kekitiinwa, Sabrina Bakeera-Kitaka, Grace Ndeezi, Robert Downing, Xiaochuan Feng, Donna E et. al. Cryptosporidiosis and Microsporidiosis in Ugandan Children with persistent Diarrhoea with and without concurrent infection with the Human Immunodefiency Virus. Am. J. Trop. Med. Hyg., 2005; 73 (5): 921-925.

[11] Joint United Nations Programme on HIV/AIDS (UNAIDS, 2002) World Health Organisation (WHO) AIDS epidemic update: UNAIDS/WHO: 2002; 2-41.

[12] Park BJ, Wannemuehler KA, Marston BJ, Govender N, Pappas PG, Chiller TM. Estimation of the current global burden of cryptococcal meningitis among persons living with HIV/AIDS. AIDS 2009; 23.

[13] Sumiti V, Mattie CP, Adam S, Carrie FB, Caleb JS, Yael B, Michael JC and Boris S. Genetic modification of the diarrheal pathogen Cryptosporidium parvum. Nature. 2015 July 23; 523 (7561): 477-480. doi: 10.1038/nature14651.

[14] Shahrokh I, Mohammad AM, Zahra GN, Mehdi A, Farzaneh M, Fatemeh N, Mehdi M, Dhammika LW, Seyed-Hossein H, Frequency and Molecular Identification of Cryptosporidium Species among Immunocompromised Patients Referred to Hospitals, Central Iran, 2015-16 Iran J Parasitol: Vol. 15, No. 1, Jan-Mar 2020, pp. 31-39.

[15] Cheesbrough M. District Laboratory Practice in Tropical Countries. Part 1, $2^{\text {nd }}$ Edition. Low Price Edition. Cambridge University Press, UK 2005; 148-56.

[16] Shahira A, Ahmed 1 and Panagiotis K. Cryptosporidium and Cryptosporidiosis: The Perspective from the Gulf Countries. Int. J. Environ. Res. Public Health 2020, 17, 6824.

[17] Wang ZD, Liu Q, Liu HH, Li S, Zhang L, Zhao YK, Zhu XQ. Prevalence of Cryptosporidium, microsporidia and Isospora infection in HIV-infected people: a global systematic review and meta-analysis. Parasit Vectors. 2018; 28 (1): 9-11.

[18] Adesiji YO, Lawal RO, Taiwo SS, Fayemiwo SA, Adeyeba OA. Cryptosporidiosis in HIV Infected Patients with Diarrhoea in Osun State, Southwestern Nigeria. Eur J Gen Med 2007; 4 (3): 119-122.

[19] Awogun IA. The prevalence of intestinal parasitic infection in Children living in Ilorin Kwara State, Nigeria. West Africa J. Med. 1984; 4 (1): 16-21.

[20] Oyerinde JPO, Adegbete-Hochist AF, Ogunbiyi O. Prevalence of intestinal parasites of man in the metropolitan Lagos. Nig. J. Nat Sc. 1979; 3: 147-155.

[21] Okodua M, Adeyeba OA, Atatfeng YM, Okpala HO. Age and sex distribution of intestinal parasitic infection among HIV infected subjects in Abeakuta, Nigeria. Online J Health Allied Scs 2003; 4:3.

[22] Fisseha, B, Petros B, Woldemchal T. Cryptosporidium and other parasites in Ethiopia AIDS patients with chronic diarrhea. East Afr Med. J. 1998; 75 (2): 100-101.

[23] Pape JW, Verdier RI, Boncy M, Boncy J, Johnson WD. Jr. Cyclospora infection in adults infected with HIV. Clinical manifestations, treatment, and prophylaxis. Ann Intern Med. 1994; 121 (9): 654-7. 